\title{
SPONTANEOUS REMISSION OF A BONY METASTASIS IN PROSTATIC ADENOCARCINOMA
}

\author{
J. R. SCHURMANS, B. G. BLIJENBERG, G. H. MICKISCH AND F. H. SCHRÖDER \\ From the Departments of Urology and Clinical Chemistry Laboratory, Academic Hospital and Erasmus University, Rotterdam,
} The Netherlands

KEY WORDS: prostate; adenocarcinoma; remission, spontaneous; neoplasm metastasis; prostatectomy

Today prostatic adenocarcinoma is the most commonly diagnosed malignancy in men. Organ confined tumor is curable but once metastasis occurs tumor progression can only be delayed temporarily.

\section{CASE REPORT}

A 59-year-old man originally presented with T3 pT3N1M0 grade 2 prostatic adenocarcinoma after radical retropubic prostatectomy. After 9 years he underwent surgical castration for a painful solitary metastatic lesion in the right pelvis, confirmed by highly elevated prostatic acid phosphatase and prostate specific antigen (PSA), and a new metastasis seen on a bone scan of the right os ischium. Palliative irradiation was given 2 years later due to increasing pain and confirmed histological evidence of metastatic prostatic adenocarcinoma tissue by bone biopsy (fig. 1). (The Tandem-E PSA assay was used before January 1993 and the Abbott IMx assay was used thereafter.) PSA increased dramatically 1 year later without clinical signs of local recurrence or new metastatic lesions. Subsequently PSA suddenly and unexpectedly decreased (fig. 2). Presently PSA has been stable at $0.4 \mu \mathrm{g} /$. (normal 0 to 4 ) for 12 months and the metastasis seen on the bone scan has completely disappeared.

\section{DISCUSSION}

Even when values are within the normal range, increasing PSA in 6 to 12 months in a hormone treated patient signals clinical progression for 80 to $85 \%$ of this population. ${ }^{1}$ Six years after surgical castration and 4 years after palliative irradiation PSA increased in our case, indicating progressive disease. The sudden decrease in PSA and complete disappearance of the metastasis from the bone scan cannot be explained by previous medical intervention. Remission of metastatic lesions after treatment of the primary prostatic tumor due to a cryosurgically induced immune response has

\section{Accepted for publication July 21, 1995.}

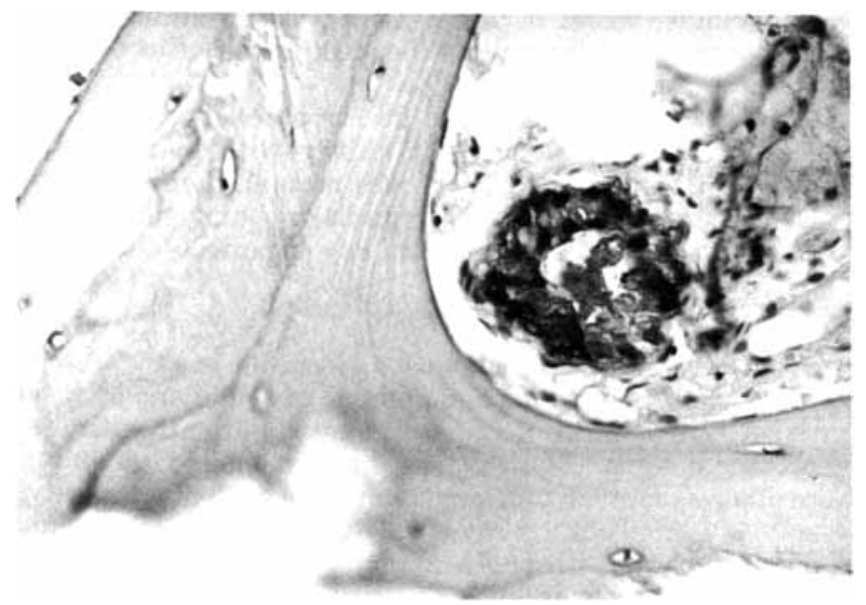

FIG. 1. Metastatic lesion in right os ischium after positive PSA staining. Reduced from $\times \mathbf{4 0 0}$.

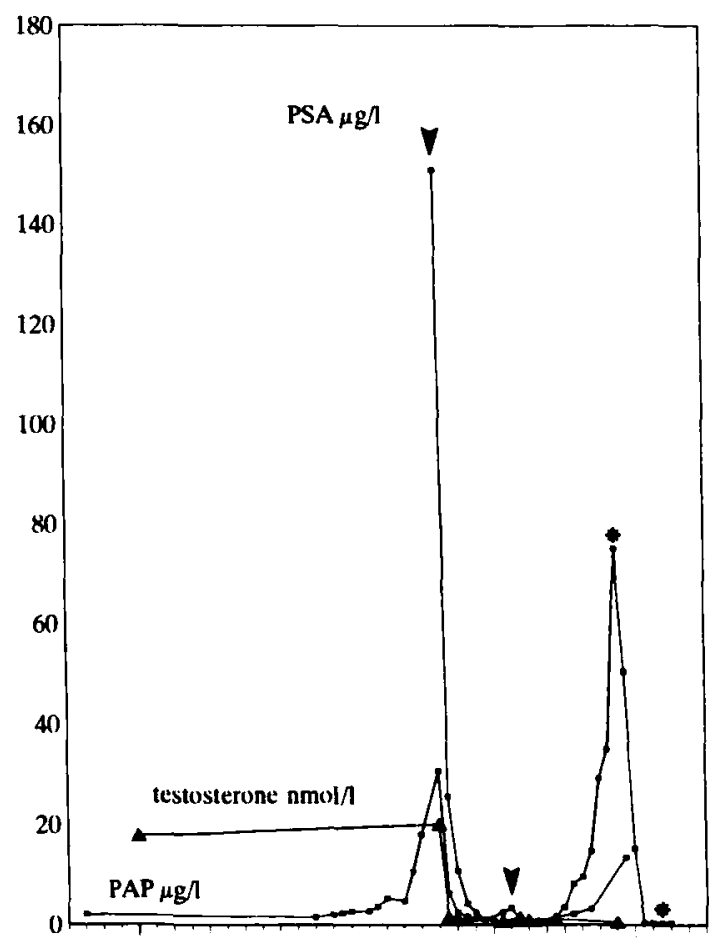

$\begin{array}{llllllllll}1978 & 1980 & 1982 & 1984 & 1986 & 1988 & 1990 & 1992 & 1994 & 1996\end{array}$

\section{time (years)}

Fig. 2. Fluctuation of serum PSA, serum prostatic acid phosphatase $(P A P)$ and serum testosterone with time. Increase in PSA was not paralleled by serum testosterone increase, excluding surreptitious testosterone intake as cause of this phenomenon. Note time of surgical castration in August 1988 (arrowhead) and palliative irradiation in June 1990 (arrowhead). PSA determinations were repeated with 4 different PSA kits $\left(^{*}\right)$.

been previously reported. ${ }^{2}$ Repeat PSA determinations with 4 kits (Hybritech, Boehringer, Wallace and Abbott) were not significantly different (fig. 2). Hyperglobulinemia, human anti-mouse antibodies or other heterophilic antibodies were unlikely to be responsible for detection errors., ${ }^{1,3}$ A literature search from 1966 to December 1994 did not reveal a similar case. Therefore, we believe that our report represents the first case of spontaneous remission of a bony metastasis of prostatic adenocarcinoma.

\section{REFERENCES}

1. Vessella, R. L. and Lange, P. H.: Issues in the assessment of PSA immunoassays. Urol. Clin. N. Amer., 20: 607, 1993.

2. Soanes, W. A., Ablin, R. J. and Gonder, M. J.: Remission of metastatic lesions following cryosurgery in prostatic cancer: immunologic considerations. J. Urol., 104: 154, 1970.

3. Slota, B. P., Magic, B. D. and Huhn, O.: Detection of a circulating immune complex of PSA and IgG in a patient with benign prostatic hyperplasia and hypergammaglobulinemia. Read at meeting of International Society for Oncodevelopmental Biology and Medicine, Gröningen, The Netherlands, September 1994. 